Dig Surg 1995;12:362

\title{
Acknowledgement to the Reviewers
}

The editors wish to thank the following reviewers for help in evaluating the manuscripts for Digestive Surgery:

John Alexander-Williams, Birmingham (UK)

Roland Andersson, Lund (Sweden)

S. Argov, Haifa (Israel)

A.R. Baker, Bristol (UK)

Lawrence S. Bizer, Bronx (USA)

U. Blum, Freiburg i.Br. (Germany)

Kurt Borch, Linkoeping (Sweden)

D. Bouchier-Hayes, Dublin (Ireland)

Peter Buchmann, Zurich (Switzerland)

Markus W. Büchler, Bern (Switzerland)

F.C. Campbell, Dundee (UK)

Isidore Cohn, New Orleans (USA)

F.E. Eckhauser, Ann Arbor (USA)

C. Ekelund, Malmö (Sweden)

Harold Ellis, London (UK)

S.T. Fan, Hong Kong (Hong Kong)

W. Ferstl, Freiburg i.Br. (Germany)

Charles F. Frey, Sacramento (USA)

Peter Funch-Jensen, Hvidovre (Denmark)

Susan Galandiuk, Louisville (USA)

O.J. Garden, Edinburgh (UK)

Jan P. Geerdsen, Koge (Denmark)

J.H. Hasse, Freiburg i.Br. (Germany)

R.U. Häring, Freiburg i.Br. (Germany)

A. Hirner, Bonn Venusberg (Germany)

M. Hollands, Westmead (Australia)

K.W. Jauch, München (Germany)

H.E. Jensen, Klampenborg (Denmark)

Raymond J. Joehl, Chicago (USA)

R. Scott Jones, Charlottesville (USA)

Th. Junginger, Mainz (Germany)

H. Keller, Freiburg i.Br. (Germany)

Günter Kirste, Freiburg i.Br. (Germany)

J.H.C. Kuijpers, Nijmegen (The Netherlands)

Gerald M. Larson, Louisville (USA)

Hans Lippert, Magdeburg (Germany)

John Miles Little, Westmead (Australia) 
Lars Lundell, Göteborg (Sweden)

Bernd C . Manegold, Mannheim (Germany)

Kurt Meissner, Tamsweg (Austria)

Antonio Mendes de Almeida, Lisbon (Portugal)

H.-J. Meyer, Hannover (Germany)

E. Moreno-Gonzales, Madrid (Spain)

Sean Mulvihill, San Francisco (USA)

W.R. Murray, Glasgow (UK)

John P. Neoptolemos, Birmingham (UK)

Gabriele Nöldge, Freiburg i.Br. (Germany)

G. Otto, Heidelberg (Germany)

J.H. Pemberton, Rochester (USA)

S.F. Phillips, Rochester (USA)

Howard A. Reber, Los Angeles (USA)

Marcel J. Rheault, Montreal (Canada)

L.F. Rikkers, Omaha (USA)

M. Rössle, Freiburg i.Br. (Germany)

G. Ruf, Freiburg i.Br. (Germany)

Klaus D. Rückauer, Freiburg i.Br. (Germany)

Jonathan Sackier, San Diego (USA)

Michael J. Sarr, Rochester (USA)

Peter M. Schlag, Berlin (Germany)

P.F. Schofield, Manchester (UK)

U. Schöffel, Freiburg i.Br. (Germany)

Ifat A. Shah, Phoenix (USA)

Rune Sjödahl, Linköping (Sweden)

Nib Soehendra, Hamburg (Germany)

Joachim Sontheimer, Freiburg i.Br. (Germany)

Jon Arne Soreide, Stavanger (Norway)

Odd Soreide, Oslo (Norway)

Arnulf Thiede, Würzburg (Germany)

J.S. Thompson, Omaha (USA)

K.H. Treutner, Aachen (Germany)

D. Tweedle, Manchester (UK)

Asgaut Viste, Bergen (Norway)

Andrews L. Warshaw, Boston (USA)

Th. Wobbes, Nijmegen (The Netherlands)

John Wood, Kansas City (USA)

Michael Zinner, Boston (USA)

362 